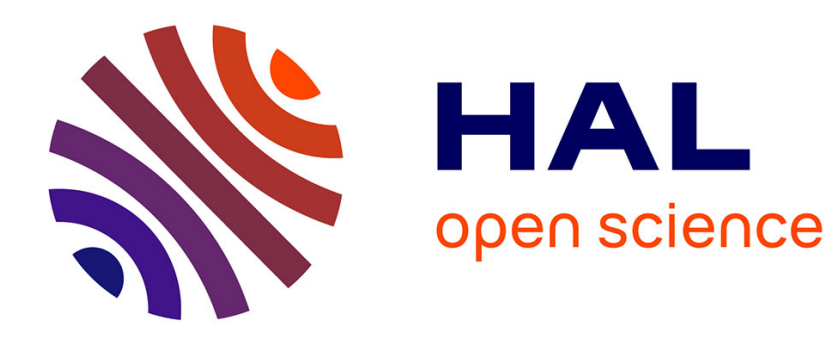

\title{
Onset of flow in a vibrated thin viscoplastic layer
} Ian Frigaard, Chérif Nouar

\section{To cite this version:}

Ian Frigaard, Chérif Nouar. Onset of flow in a vibrated thin viscoplastic layer. Journal of NonNewtonian Fluid Mechanics, 2019, 10.1016/j.jnnfm.2019.02.009 . hal-02408902

\section{HAL Id: hal-02408902 \\ https://hal.science/hal-02408902}

Submitted on 13 Dec 2019

HAL is a multi-disciplinary open access archive for the deposit and dissemination of scientific research documents, whether they are published or not. The documents may come from teaching and research institutions in France or abroad, or from public or private research centers.
L'archive ouverte pluridisciplinaire HAL, est destinée au dépôt et à la diffusion de documents scientifiques de niveau recherche, publiés ou non, émanant des établissements d'enseignement et de recherche français ou étrangers, des laboratoires publics ou privés. 


\title{
Onset of flow in a vibrated thin viscoplastic layer
}

\author{
Ian Frigaard ${ }^{a}$, Cherif Nouar ${ }^{b}$ \\ ${ }^{a}$ Department of Mathematics and Department of Mechanical Engineering, \\ University of British Columbia, 2324 Main Mall, Vancouver, BC, Canada, V6T \\ $1 Z 4$. \\ ${ }^{\mathrm{b}}$ UMR 7563, CNRS - Université de Lorraine, 1\&4, avenue de la Fort de Haye, \\ TSA 60604, 54518 Vandoeuvre-Les-Nancy Cedex, France.
}

\begin{abstract}
The onset of flow in vibrated layer of viscoplastic fluid is investigated theoretically, using a lubrication approximation. The rheological behavior of the fluid is described by the Herschel-Bulkley model. The equation describing the evolution of the free surface is derived. Four different regimes are found depending on the ratio $\chi$ of the gravitational acceleration to the acceleration of vibration and the ratio of the yield stress to the shear-stress at the vibrated wall. Furthermore, a necessary condition for instability is derived. The different regimes are illustrated in the case of a 1D problem.
\end{abstract}

Key words: Viscoplastic fluids, Faraday

\section{Introduction}

When a horizontal fluid layer is subjected to vertical oscillations, its free surface becomes unstable to standing waves beyond a certain threshold. This phenomenon was first observed by Faraday [1]. He, noticed that the vibrating frequency of the waves is half of that of the forcing. The onset of these waves was first determined by Benjamin and Ursell [2] for an incompressible inviscid fluid. Using linear stability analysis, they showed that for an inviscid infinite layer of fluid, the onset problem reduces to analysis of Mathieu's equation, which allows harmonic and subharmonic responses. Kumar and Tuckerman [3] extended the linear stability analysis of Benjamin and Ursell [2] to viscous fluids. In the last few decades, several studies have been devoted to the Faraday instability in Newtonian fluids and focused on pattern formation. According to the driving frequency and amplitude and the fluid viscosity, the free 
surface may exhibit stripes, squares, hexagons and quasi-crystalline patterns; see e.g. [4-9].

Recently, interest has grown in the effect of vertical vibrations on a layer of a complex fluid both theoretically and experimentally. Raynal et al. [10] provided the first experimental data for polymer solutions. Their work concentrates on dilute polymer solutions where the influence of elasticity is small. They found a slight shift of the critical accelerations; the critical wave numbers were not affected. Muller \& Zimmermann [11] presented a linear stability analysis for a Maxwell fluid. They found that when the inverse of the relaxation time compares to the driving frequency, a harmonic response of the fluid surface might become unstable rather than the classical subharmonic response. The signature of elasticity in the Faraday instability was demonstrated by Ballesta \& Manneville [12] using a wormlike micelle solution. They observed that the critical accelerations and wavenumbers present oscillations as a function of driving frequency and fluid height. For shear-thickening fluids, an interesting phenomenon was discovered by Merkt et al. [13]. Using a cornstarch and glass micro-sphere suspension, the authors found that holes which were created by applying a finite amplitude perturbation to the fluid layer were persistent in a certain range of frequency and acceleration. The stabilizing mechanism of persistent holes is currently unknown. Merkt et al. [13] argued that the increase of the viscosity with the applied shear stress is the key factor. Deegan [14] suggested a different mechanism, independent of whether the fluid is shear-thinning or shear-thickening, based on stress hysteresis. The class of complex fluids that support persistent holes was widened to emulsions by Falcon et al. [15]. Thus, it seems that shear-thickening is unnecessary for these structures to form, contrary to initial claims [13].

Here we consider the case of viscoplastic fluids, i.e. fluids that exhibit yield stress behaviour. A comprehensive review of the physics of yield stress materials and how it is related to the microstructure was recently presented by Bonn et al. [16] for a broad range of materials (emulsion, gels, colloidal suspensions, ...). Using a polymeric gel (ultrasound gel Dane-gel R1) with a significant yield stress $(146 \pm 6 \mathrm{~Pa})$, Shiba et al. [17] observed a new pattern which consists in a pair of counter rotating vortices. Qualitatively, similar results were also obtained using shaving gel or toothpaste. To characterize the onset of convection, Shiba et al. [17] introduced a non-dimensional parameter $\Sigma$, representing the ratio of the stress exerted by the plate due to inertia of the material, divided by the yield stress. The onset of convection occurred for $\Sigma=O(1)$. The convective rolls appear if the blob of gel is either placed directly on a horizontal plate without sidebands, or within a vertical oscillating cylinder. The list of complex fluids for which persistent holes can be observed, when they are submitted to vertical mechanical vibration, increased further after the experiments of Wolf et al. [18]. The authors used Carbopol gel (a high molecular weight, hydrophilic, and crosslinked polyacrylic acid polymer) as a 
test fluid, reporting yield stresses in the range $34-184 \mathrm{~Pa}$ (depending on the measurement and fitting method).

There are two main contributions in [18]. Experimentally, the authors observed the formation of persistent holes in a regular geometric pattern in the surface of initially circular discs of Carbopol solutions, subjected to vertical oscillations of varying frequency and amplitude. Here the hole patterns are formed within an intermediate linearly increasing band of acceleration versus frequency. Above this band, they report behaviour analogous to [17], although not explored here, and below the band no flow occurred. Within the band, increasing oscillation frequency produced an increasing number of modes in the patterns which could be stably observed and which grew eventually into persistent holes within the sample. In sense therefore, the study of [18] can be thought of as a more in depth study of the transition of [17], which appears not to be a sharp transition to the roll-structure, but instead has a rich intermediate patterning structure.

It is hard to interpret the effects of the vertical vibration directly on the rheology, in this type of experiment. Yield stress behaviours arise in many different materials [16] and depend on the structure of the material. The application of a vertical vibration to certain types of yield stress materials may modify significantly the internal structure leading to a strong reduction of the yield stress. This has been observed for concrete by Tattersall \& Baker [19] and for highly concentrated suspension of milk chocolate by Ouriev \& Uriev [20]. Carbopol is not however a suspension and this type of multiphase mechanism is probably not present. On the other hand, rheological properties of Carbopol solutions are strongly dependent on the type of Carbopol used, its concentration and the preparation method. Elastic effects are certainly present at low shear, although there is no suggestion that the onset in [18] is due to elasticity in the same sense as [12]. Thixotropic hysteresis has also been observed for some Carbopol solutions. If this provides a rheology reduction mechanism for these experiments is less clear: many thixotropic models require a strain rate or viscous dissipation to initiate destruction, i.e. flow is needed for destruction. If however there is a reduction in yield stress, it is still noticeable that there appears to be a yield-stress dependent onset threshold in the experiments of $[17,18]$, below which there is no motion. Thus, onset of motion for a fluid with a yield stress is of interest.

The second part of [18] presents a form of lubrication flow analysis. The final form of evolution equation for the surface is a parabolic equation in which an acceleration dependent viscosity is made separable from the horizontal Laplacian operator. This separable structure evidently leads to a modal solutions and the authors are able to produce qualitatively similar patterns to their experiments. Two aspects of [18] are however unsatisfactory in our opinion. First, having studied a material that is clearly non-Newtonian the authors 
commence by assuming the fluid is Newtonian, but then later introduce an unspecified dependency of the viscosity on the acceleration. Both points are obscure. Second, the linearized equations appear to assume that the fluids are always flowing, which means that the issue of onset in terms of how the yield stress is overcome is not addressed, i.e. this is pattern formation in a yielded fluid. Indeed the role of the yield stress is unclear in the model although the experimental study reports significant regimes of no flow. While the pattern formation aspects are of interest, this leaves the question of flow/no-flow untreated.

In this paper we develop a thin-film/lubrication approach and model only flow onset in a Faraday setup. The thin-film approach is classical and results in a model (different to that in [18]) in which the acceleration acts via the surface gradients to induce horizontal motion in the flow, i.e. film spreading. We discuss the scaling under which this mode takes place, the different qualitative behaviours that result and the potential onset of instability. The aim of this short paper is simply to show what results from this style of analysis and the assumptions of an ideal visco-plastic fluid model. The behaviours predicted are somewhat different to the experiments of $[17,18]$, although of course the model situation is also different. We end the paper by discussing some of the challenges to improving this.

\section{Governing equations}

We consider a layer of an incompressible yield stress fluid on a horizontal plate which is subjected to a vertical sinusoidal oscillation of amplitude $\hat{a}$ and a frequency $\hat{f}$. The mean depth of the fluid layer is denoted $\hat{h}_{0}$. In the frame of reference which moves with the oscillating plate, the gravitational acceleration $\hat{\boldsymbol{G}}$ is temporarily modulated,

$$
\hat{\boldsymbol{G}}=-(\hat{g}-\hat{a} \cos \hat{\omega} \hat{t}) \boldsymbol{e}_{z}
$$

Here, $\boldsymbol{e}_{z}$ is the unit-vector in the vertical $z$-direction, and $\hat{\omega}=2 \pi \hat{f}$. The fluid satisfies the continuity and momentum equations:

$$
\begin{aligned}
\hat{\boldsymbol{\nabla}} \cdot \hat{\boldsymbol{u}} & =0, \\
\hat{\rho} \frac{D}{D \hat{t}} \hat{\boldsymbol{u}} & =-\hat{\nabla} \hat{p}+\hat{\nabla} \cdot \hat{\boldsymbol{\tau}}+\hat{\rho} \hat{\boldsymbol{G}},
\end{aligned}
$$

where $\hat{\boldsymbol{u}}$ denotes the velocity, $\hat{p}$ is the pressure and $\hat{\boldsymbol{\tau}}$ is the deviatoric stress tensor, Throughout the paper we shall adopt the convention of denoting dimensional quantities and variables with the "hat" symbol, i.e. $\hat{\ominus}$ 
We assume that the rheological behavior of the yield stress fluid is described by the Herschel-Bulkley model:

$$
\begin{cases}\hat{\boldsymbol{\tau}}=\left(\hat{\kappa} \hat{\dot{\gamma}}(\hat{\boldsymbol{u}})^{n-1}+\frac{\hat{\tau}_{y}}{\hat{\dot{\gamma}}(\hat{\boldsymbol{u}})}\right) \hat{\dot{\gamma}}(\hat{\boldsymbol{u}}), & \text { if } \hat{\tau}>\hat{\tau}_{y} \\ \hat{\dot{\gamma}}(\hat{\boldsymbol{u}})=0 & \text { if } \hat{\tau} \leq \hat{\tau}_{y}\end{cases}
$$

where $\hat{\tau}_{y}, \hat{\kappa}$ and $n$ are the yield stress, consistency and power-law index of the fluid, respectively. The tensor $\hat{\dot{\gamma}}(\hat{\boldsymbol{u}})$ is the rate of strain tensor associated with the velocity field $\hat{\boldsymbol{u}}$, defined component wise as

$$
\hat{\dot{\gamma}}_{i j}(\hat{\boldsymbol{u}}):=\frac{\partial \hat{u}_{i}}{\partial \hat{x}_{j}}+\frac{\partial \hat{u}_{j}}{\partial \hat{x}_{i}}, \quad(\hat{u}, \hat{v}, \hat{w})=\left(\hat{u}_{1}, \hat{u}_{2}, \hat{u}_{3}\right),(\hat{x}, \hat{y}, \hat{z})=\left(\hat{x}_{1}, \hat{x}_{2}, \hat{x}_{3}\right) .
$$

$\hat{\dot{\gamma}}$ and $\hat{\tau}$ are norms of $\hat{\dot{\gamma}}$ and $\hat{\boldsymbol{\tau}}$, defined as

$$
\hat{\dot{\gamma}}=\sqrt{\frac{1}{2} \sum_{i j} \hat{\dot{\gamma}}_{i j}^{2}} \quad \text { and } \quad \hat{\tau}=\sqrt{\frac{1}{2} \sum_{i j} \hat{\tau}_{i j}^{2} .}
$$

For boundary conditions, on the plate surface the no-slip and no-penetration boundary conditions take the form:

$$
\hat{\boldsymbol{u}}=0 \quad \text { at } \hat{z}=-\hat{h}_{0}
$$

The free surface of the fluid layer is at $\hat{z}=\hat{\xi}(\hat{x}, \hat{y}, \hat{t})$, where $\hat{\xi}$ has zero mean, i.e. $\hat{h}_{0}$ is the mean depth of the fluid layer. The motion of the free surface is governed by the kinematic condition:

$$
\frac{\partial \hat{\xi}}{\partial \hat{t}}+\hat{\boldsymbol{u}}_{H} \cdot \hat{\nabla}_{H} \hat{\xi}=\hat{w}
$$

where $\hat{\boldsymbol{u}}_{H}$ and $\hat{\nabla}_{H}$ denote the horizontal components of the velocity and divergence operator, respectively. At the interface between air and fluid, the diference between stresses is balanced by surface tension:

$$
\left[\hat{\boldsymbol{\sigma}}^{a}-\hat{\boldsymbol{\sigma}}\right] \cdot \boldsymbol{n}=\hat{\gamma}_{s}[\hat{\boldsymbol{\nabla}} \cdot \boldsymbol{n}] \boldsymbol{n}, \quad \text { at } \quad \hat{z}=\hat{\xi}
$$

where $\hat{\boldsymbol{\sigma}}^{a}=-\hat{p}^{a} \boldsymbol{\delta}, \hat{p}^{a}$ is the atmospheric pressure, $\hat{\boldsymbol{\sigma}}=-\hat{p} \boldsymbol{\delta}+\hat{\boldsymbol{\tau}}$, and

$$
\boldsymbol{n}=\frac{\left(-\frac{\partial \hat{\xi}}{\partial \hat{x}},-\frac{\partial \hat{\xi}}{\partial \hat{y}}, 1\right)}{\left(1+\left(\frac{\partial \hat{\xi}}{\partial \hat{x}}\right)^{2}+\left(\frac{\partial \hat{\xi}}{\partial \hat{y}}\right)^{2}\right)^{1 / 2}}
$$


Denoting by $\boldsymbol{t}$ any tengential vector perpendicular to $\boldsymbol{n}$, the dot product of (9) with $\boldsymbol{t}$ leads to

$$
\boldsymbol{t} \cdot(\hat{\boldsymbol{\tau}} \cdot \boldsymbol{n})=0
$$

In the following, we assume that the amplitude of the free-surface deformation is much smaller than the characteristic length of the horizontal layer:

$$
\left\|\nabla_{H} \hat{\xi}\right\|<<1
$$

Combining (10) with (11) shows that the tangential stress components vanish,

$$
\hat{\tau}_{x z}=0 \quad \text { and } \quad \hat{\tau}_{y z}=0 \text { at } \hat{z}=\hat{\xi}
$$

\subsection{Stationary layers}

Before proceeding, let us examine the stationary state that we expect to exist for sufficiently small oscillations. We assume that $\hat{\boldsymbol{u}}=0$ and that $\hat{\xi}$ is independent of $\hat{t}$. The momentum equations reduce to:

$$
0=-\hat{\nabla} \hat{p}+\hat{\nabla} \cdot \hat{\boldsymbol{\tau}}+\hat{\rho} \hat{\boldsymbol{G}}
$$

and for the moment lets assume that $\hat{\boldsymbol{\tau}}=0$. Thus, the pressure is given by:

$$
\begin{aligned}
\hat{p} & =\hat{p}^{a}+\hat{\gamma}_{s}[\hat{\boldsymbol{\nabla}} \cdot \boldsymbol{n}], \text { at } \hat{z}=\hat{\xi}(\hat{x}, \hat{y}), \\
\hat{p}(\hat{x}, \hat{y}, \hat{z}) & =\hat{p}^{a}+\hat{\gamma}_{s}[\hat{\boldsymbol{\nabla}} \cdot \boldsymbol{n}]+\hat{\rho}[\hat{g}-\hat{a} \cos \hat{\omega} \hat{t}][\hat{\xi}(\hat{x}, \hat{y})-\hat{z}]
\end{aligned}
$$

We observe that horizontal pressure gradients now exist within the fluid layer:

$$
\hat{\nabla}_{H} p(\hat{x}, \hat{y}, \hat{z})=\hat{\nabla}_{H}\left(\hat{\gamma}_{s}[\hat{\boldsymbol{\nabla}} \cdot \boldsymbol{n}]+\hat{\rho}[\hat{g}-\hat{a} \cos \hat{\omega} \hat{t}] \hat{\xi}\right),
$$

induced by horizontal gradients in elevation and surface curvature.

Evidently, (13) cannot be satisfied in the absence of deviatoric stresses within the layer, i.e. $\hat{\tau} \neq 0$. This does not invalidate the potential for a stationary layer, but it is necessary that the deviatoric stresses lie below the yield stress of the fluid layer, i.e. and consequently that there is a non-zero yield stress. In general the stresses are indeterminate in this situation. The above analysis 
serves to indicate the magnitude of the deviatoric stresses induced by horizontal gradients. As in all Faraday type problems the deviatoric stresses come from gradients in $\hat{\xi}$, both in the surface tension and spreading terms within (16). With $\hat{\nabla}_{H} \hat{\xi}=0$, we should expect no motion at all in any ideal viscoplastic fluid, i.e. regardless of $|\hat{G}|$. Furthermore, for a given size of horizontal surface gradient we can make a preliminary estimate of the required yield stress to prevent motion.

\subsection{Dimensionless setting}

Let us suppose that the initial surface profile $\hat{\xi}$ has maximal amplitude $\hat{\xi}_{0}$ and varies over a horizontal length-scale $\hat{L}_{0}$. We shall make the assumption that $\delta=\hat{h}_{0} / \hat{L}_{0} \ll 1$ and seek to develop a thin-film style of model. We assume that lengths and velocities scale as:

$$
(\hat{u}, \hat{v}, \hat{w})=\hat{U}_{0}(u, v, \delta w), \quad(\hat{x}, \hat{y}, \hat{z})=\hat{L}_{0}(x, y, \delta z), \quad \hat{t}=\frac{\hat{L}_{0}}{\hat{U}_{0}} t
$$

and write $\hat{\xi}=\hat{h}_{0} \xi(x, y, t)$ and $\hat{\xi}_{0}=\hat{h}_{0} \xi_{0}$.

The static layer analysis suggests that the pressure consists of 2 components: a time varying (static) part and a component due to the surface profile. Accordingly define the dimensionless pressure $p$ via:

$$
\hat{p}=\hat{p}^{a}-\hat{\rho}[\hat{g}-\hat{a} \cos \hat{\omega} \hat{t}] \hat{z}+\hat{\rho}[\hat{g}+\hat{a}] \hat{\xi}_{0} p .
$$

Here we have implicitly taken the buoyant component of pressure rather than the surface tension as the dominant driving force. We observe that the maximal horizontal pressure gradients should scale approximately like:

$$
\left|\hat{\nabla}_{H} p\right| \sim O\left(\frac{\hat{\xi}_{0} \hat{\rho}(\hat{g}+\hat{a})}{\hat{L}_{0}}\right)\left[1+O\left(\frac{\hat{\gamma}_{s}}{\hat{\rho}(\hat{g}+\hat{a}) \hat{L}_{0}^{2}}\right)\right] .
$$

Assuming a balance of the horizontal pressure gradients primarily through shear stress gradients across the depth of the fluid layer, this suggests that shear stresses imposed on the fluid layer should scale like:

$$
|\hat{\boldsymbol{\tau}}| \sim O\left(\frac{\hat{h}_{0} \hat{\xi_{0}} \hat{\rho}(\hat{g}+\hat{a})}{\hat{L}_{0}}\right)\left[1+O\left(\frac{\hat{\gamma}_{s}}{\hat{\rho}(\hat{g}+\hat{a}) \hat{L}_{0}^{2}}\right)\right] .
$$

These imposed stresses are opposed by the yield stress of the fluid and by viscous stresses. We define the velocity scale $\hat{U}_{0}$ by: 


$$
\hat{U}_{0}=\hat{h}_{0}\left(\frac{\hat{h}_{0} \hat{\xi}_{0} \hat{\rho}(\hat{g}+\hat{a})}{\hat{L}_{0} \hat{\kappa}}\right)^{1 / n}
$$

and use this to define a typical viscous scale $\hat{\tau}_{0}$ :

$$
\hat{\tau}_{0} \equiv \hat{\kappa}\left(\frac{\hat{U}_{0}}{\hat{h}_{0}}\right)^{n}=\frac{\hat{h}_{0} \hat{\xi}_{0} \hat{\rho}(\hat{g}+\hat{a})}{\hat{L}_{0}} .
$$

The Bingham number $B$ is defined as:

$$
B=\frac{\hat{L}_{0} \hat{\tau}_{y}}{\hat{h}_{0} \hat{\xi}_{0} \hat{\rho}(\hat{g}+\hat{a})}=\frac{\hat{\tau}_{y}}{\hat{\tau}_{0}}
$$

which is observed to be the ratio of yield stress to buoyancy stress, or alternatively yield to viscous stress. Although strictly speaking the stress scaling is derived in order to balance the principal shear stresses, many studies have shown that long-thin geometries of yield stress fluids are characterized by pseudo-plug regions in which the extensional components of $\hat{\boldsymbol{\tau}}$ have similar magnitude to the shear components. Thus, we assume that

$$
\begin{gathered}
\hat{\tau}_{z x}=\hat{\tau}_{x z} \sim \hat{\tau}_{0}, \quad \hat{\tau}_{z y}=\hat{\tau}_{y z} \sim \hat{\tau}_{0}, \quad \hat{\tau}_{y x}=\hat{\tau}_{x y} \sim \delta \hat{\tau}_{0}, \\
\hat{\tau}_{x x} \sim \hat{\tau}_{0}, \quad \hat{\tau}_{y y} \sim \hat{\tau}_{0}, \quad \hat{\tau}_{z z} \sim \hat{\tau}_{0},
\end{gathered}
$$

noting that in fully yielded fluid regions the extensional stresses would drop to $O(\delta)$.

The scaled momentum equations are

$$
\begin{aligned}
\delta R e \frac{D}{D t}(u, v) & =-\nabla_{H} p+\frac{\partial}{\partial z}\left[\tau_{x z}, \tau_{y z}\right]+O(\delta), \\
\delta^{3} \operatorname{Re} \frac{D}{D t} w & =-\frac{\partial p}{\partial z}+O(\delta),
\end{aligned}
$$

where the Reynolds number $R e$ is defined by:

$$
R e=\frac{\hat{L}_{0}^{2}}{\hat{\xi}_{0}(\hat{g}+\hat{a})}\left(\frac{\hat{h}_{0} \hat{\xi}_{0} \hat{\rho}(\hat{g}+\hat{a})}{\hat{L}_{0} \hat{\kappa}}\right)^{2 / n}, \quad f=\frac{\hat{L}_{0} \hat{\omega}_{0}}{2 \pi \hat{U}_{0}} .
$$

The neglected terms in (21) \& (22) correspond to the next largest deviatoric stress terms. Thus, even with the assumed scaling of the extensional stresses they still remain absent in the leading order problem, as $\delta \rightarrow 0$ at fixed $R e$ : 


$$
\begin{aligned}
\nabla_{H} p & =\frac{\partial}{\partial z}\left[\tau_{x z}, \tau_{y z}\right] \\
\frac{\partial p}{\partial z} & =0
\end{aligned}
$$

From (24) we see that the left-hand side of (23) is independent of $z$ and hence integrate (24) to give:

$$
\left[\tau_{x z}, \tau_{y z}\right]=[z-\xi(x, y)] \nabla_{H} p
$$

In regions where the fluid is yielded the velocity scaling implies that the largest strain rates are

$$
\dot{\gamma}_{x z} \sim \frac{\partial u}{\partial z}+O\left(\delta^{2}\right), \quad \dot{\gamma}_{y z} \sim \frac{\partial v}{\partial z}+O\left(\delta^{2}\right)
$$

Therefore, the leading order constitutive laws are:

$$
\begin{aligned}
\left(\tau_{x z}, \tau_{y z}\right) & =\left[\dot{\gamma}^{n-1}+\frac{B}{\dot{\gamma}}\right]\left(\dot{\gamma}_{x z}, \dot{\gamma}_{y z}\right), \quad \Leftrightarrow\left|\left(\tau_{x z}, \tau_{y z}\right)\right|>B, \\
\left(\dot{\gamma}_{x z}, \dot{\gamma}_{y z}\right) & =0, \quad \Leftrightarrow\left|\left(\tau_{x z}, \tau_{y z}\right)\right| \leq B .
\end{aligned}
$$

Here note that:

$$
\dot{\gamma}=\left[\left(\frac{\partial u}{\partial z}\right)^{2}+\left(\frac{\partial v}{\partial z}\right)^{2}\right]^{1 / 2}
$$

We observe that $\left[\tau_{x z}, \tau_{y z}\right]$ is a vector that locally points in the direction of $\nabla_{H} p$, which is independent of $z$. By orienting a local coordinate system in the direction of $-\nabla_{H} p$, say $s$, we see that the shear stress normal to $s$ is zero and hence also the velocity component. If the velocity component in the $s$-direction is denoted $v$, then we have the following $1 \mathrm{D}$ problem:

$$
\begin{aligned}
\tau_{s z} & =[\xi(x, y)-z]\left|\nabla_{H} p\right|, \\
\tau_{s z} & =\left[\left|\frac{\partial v}{\partial z}\right|^{n-1}+\frac{B}{\left|\frac{\partial v}{\partial z}\right|}\right] \frac{\partial v}{\partial z}, \quad \Leftrightarrow\left|\tau_{s z}\right|>B, \\
\frac{\partial v}{\partial z} & =0, \quad \Leftrightarrow\left|\tau_{s z}\right| \leq B .
\end{aligned}
$$

We solve for $v$ using the no-slip condition at $z=0$, and integrate across the fluid layer to find the areal flux: 


$$
\begin{aligned}
\int_{-1}^{\xi} v \mathrm{~d} z & =\frac{\left|\nabla_{H} p\right|^{1 / n}\left(1-\theta_{Y}\right)_{+}^{1+1 / n}(1+\xi)^{2+1 / n}}{2+1 / n}\left(1+\theta_{Y} \frac{1}{1+1 / n}\right), \\
\theta_{Y} & =\frac{B}{(1+\xi)\left|\nabla_{H} p\right|} .
\end{aligned}
$$

Note that $\left(1-\theta_{Y}\right)_{+}=\max \left\{0,1-\theta_{Y}\right\}$, i.e. the positive part. Physically, $\theta_{Y}$ gives the ratio of yield stress to basal shear stress (at the bottom of the fluid layer): if $\theta_{Y} \geq 1$ then $v=0$.

Returning now to the two-dimensional problem with $\left[\tau_{x z}, \tau_{y z}\right]$ in the direction of $\nabla_{H} p$, we find that:

$$
\int_{-1}^{\xi}(u, v) \mathrm{d} z=-\nabla_{H} p \frac{\left|\nabla_{H} p\right|^{1 / n-1}\left(1-\theta_{Y}\right)_{+}^{1+1 / n}(1+\xi)^{2+1 / n}}{2+1 / n}\left(1+\frac{\theta_{Y}}{1+1 / n}\right) .
$$

Combining the kinematic equation with the mass conservation equation gives:

$$
\begin{aligned}
\frac{\partial \xi}{\partial t} & =w-(u, v) \cdot \nabla_{H} \xi=-\nabla_{H} \cdot \int_{-1}^{\xi}(u, v) \mathrm{d} z+\int_{-1}^{\xi} \nabla \cdot \boldsymbol{u} \mathrm{d} z, \quad \Rightarrow \\
0 & =\frac{\partial \xi}{\partial t}+\nabla_{H} \cdot \int_{-1}^{\xi}(u, v) \mathrm{d} z .
\end{aligned}
$$

Finally, we integrate the $z$-momentum equation to find the pressure from the normal stress condition. In dimensionless form this is:

$$
p=\alpha(t) \xi-\frac{\nabla_{H}^{2} \xi}{C a}
$$

where

$$
\begin{aligned}
\alpha(t) & =\frac{[\hat{g}-\hat{a} \cos \omega t]}{[\hat{g}+\hat{a}]}, \quad \omega=\frac{\hat{\omega} \hat{L}_{0}}{\hat{U}_{0}}, \\
C a & =\frac{\hat{\rho}[\hat{g}+\hat{a}] \hat{L}_{0}^{2}}{\hat{\gamma}_{s}}=\frac{1}{\xi_{0} \delta^{2}} \frac{\hat{\gamma}_{0}}{\hat{\gamma}_{s} \hat{L}_{0}}
\end{aligned}
$$

with $C a$ denoting the capillary number. The plate oscillation is captured in the term $\alpha(t)$. We observe that

$$
\nabla_{H} p=\alpha(t) \nabla_{H} \xi-\frac{\nabla_{H}^{3} \xi}{C a}
$$

which depends on $\nabla_{H} \xi, \nabla_{H}^{3} \xi, \alpha(t)$ and $C a$. Therefore, we may write: 


$$
\int_{-1}^{\xi}(u, v) \mathrm{d} z \equiv \boldsymbol{q}\left(\xi, \nabla_{H} \xi, \nabla_{H}^{3} \xi, B, n, \alpha(t), C a\right)
$$

defined by (33). Evolution of the surface is governed by solving:

$$
\frac{\partial \xi}{\partial t}+\nabla_{H} \cdot \boldsymbol{q}\left(\xi, \nabla_{H} \xi, \nabla_{H}^{3} \xi, B, n, \alpha(t), C a\right)=0
$$

In comparison to the lubrication model in [18], we note that even neglecting the surface tension term $(C a=\infty)$ these evolution equations are quite different. Equation (40) can not be linearised about a fixed film thickness and result in the separable non-conservative form given in [18]. In particular, whether the fluid is flowing or not is governed by the term $\left(1-\theta_{Y}\right)_{+}$in (33) in which the gradient of the layer thickness appears and not the layer thickness. Indeed this feature is missing from [18] as the authors assume that their fluid is described rheologically as through a time-dependent Newtonian viscosity.

\section{1D and no surface tension}

For simplicity, for the remainder of the paper we consider the 1D model with no surface tension $C a=\infty$ :

$$
\begin{aligned}
0 & =\frac{\partial \xi}{\partial t}+\frac{\partial}{\partial x} q\left(\xi, \nabla_{H} \xi, B, n, \alpha(t)\right) \\
q & =-\alpha(t) \xi_{x} \frac{\left|\alpha(t) \xi_{x}\right|^{1 / n-1}\left(1-\theta_{Y}\right)_{+}^{1+1 / n}(1+\xi)^{2+1 / n}}{2+1 / n}\left(1+\frac{\theta_{Y}}{1+1 / n}\right) \\
\theta_{Y} & =\frac{B}{\left|\alpha(t) \xi_{x}\right|(1+\xi)} .
\end{aligned}
$$

A similar analysis could be carried out for the 1D axisymmetric version of (40) with broadly similar conclusions.

Discounting the $\alpha(t)$ in front, the divergence of $-q$ is a quasilinear elliptic operator. Thus, when $\alpha(t)>0$, (41) is a quasilinear parabolic equation, describing the (modified) gravitational spreading of the surface. This diffusive spreading is enhanced by both larger $\alpha(t)>0$ and stronger gradients in the liquid layer. We can see however that diffusive spreading approaches zero if $\theta_{Y} \rightarrow 1^{-}$, which happens as either the gradients or the acceleration $\alpha(t)$ weaken sufficiently, i.e. at a constant $\alpha(t)$ we expect that the interface evens out such that $\theta_{Y} \rightarrow 1^{-}$and the spreading stops. On the other hand, for any $\theta_{Y}<1$, if $\alpha(t)<0$, the spreading is no longer diffusive but anti-diffusive, 
i.e. small wavelengths are amplified and the surface motion is expected to become unstable. We now explore these regimes and where they occur.

\subsection{Solutions and regimes}

It helps to illustrate the motions by considering 3 example initial surface profiles, as follows:

(1) Linear profile : $\xi=2 \xi_{0}\left(\frac{1}{4}-|x|\right)$

(2) Cosine profile $\xi=\frac{1}{2} \xi_{0} \cos (2 \pi x)$

(3) Parabolic profile $\xi=4 \xi_{0}\left[x^{2}-\frac{1}{12}\right]$

Note that each profile has zero mean and the parameter $\xi_{0}$ has been chosen to represent the amplitude of the surface variation, i.e. $\xi_{0}=\xi_{\max }-\xi_{\min }$. The 3 initial conditions are displayed in Fig. 1a.

\subsubsection{Static layers}

We first consider whether or not the initial surface flows at all. The dimensionless acceleration $\alpha(t)$ is given by:

$$
\alpha(t)=\frac{\chi-\cos (2 \pi f t)}{\chi+1} \in\left[\frac{\chi-1}{\chi+1}, 1\right],
$$

where $\chi=\hat{g} / \hat{a}$. Thus, the minimal value of $\theta_{Y}$ is found when $\alpha(t)=1$ and at the position where $\left|\xi_{x}\right|(1+\xi)$ is maximal. We denote:

$$
A\left(\xi_{0}\right)=\max _{x \in[-1 / 2,1 / 2]}\left\{\left|\xi_{x}\right|(1+\xi)\right\}
$$

which we find to be:

$$
\begin{aligned}
& A\left(\xi_{0}\right)=2 \xi_{0}\left(1+\xi_{0} / 2\right), \quad \text { for the linear profile, } \\
& A\left(\xi_{0}\right)=\pi \xi_{0}\left(1+\frac{\xi_{0}}{2} \cos \left(2 \pi x^{*}\right)\right) \sin \left(2 \pi x^{*}\right), \quad \text { for the cosine profile, } \\
& A\left(\xi_{0}\right)=4 \xi_{0}\left(1+\frac{2}{3} \xi_{0}\right), \quad \text { for the parabolic profile. }
\end{aligned}
$$

Here $x^{*}$ is solution of

$$
\cos \left(2 \pi x^{*}\right)+\frac{\xi_{0}}{2} \cos \left(4 \pi x^{*}\right)=0
$$


(a)

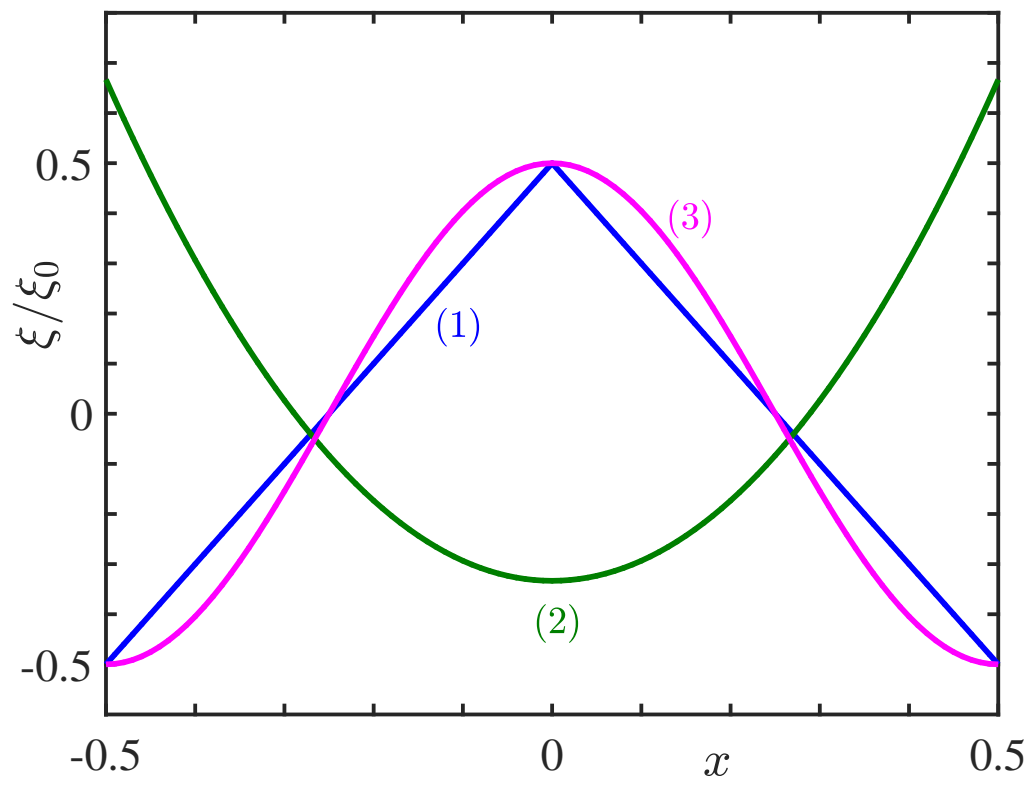

(b)

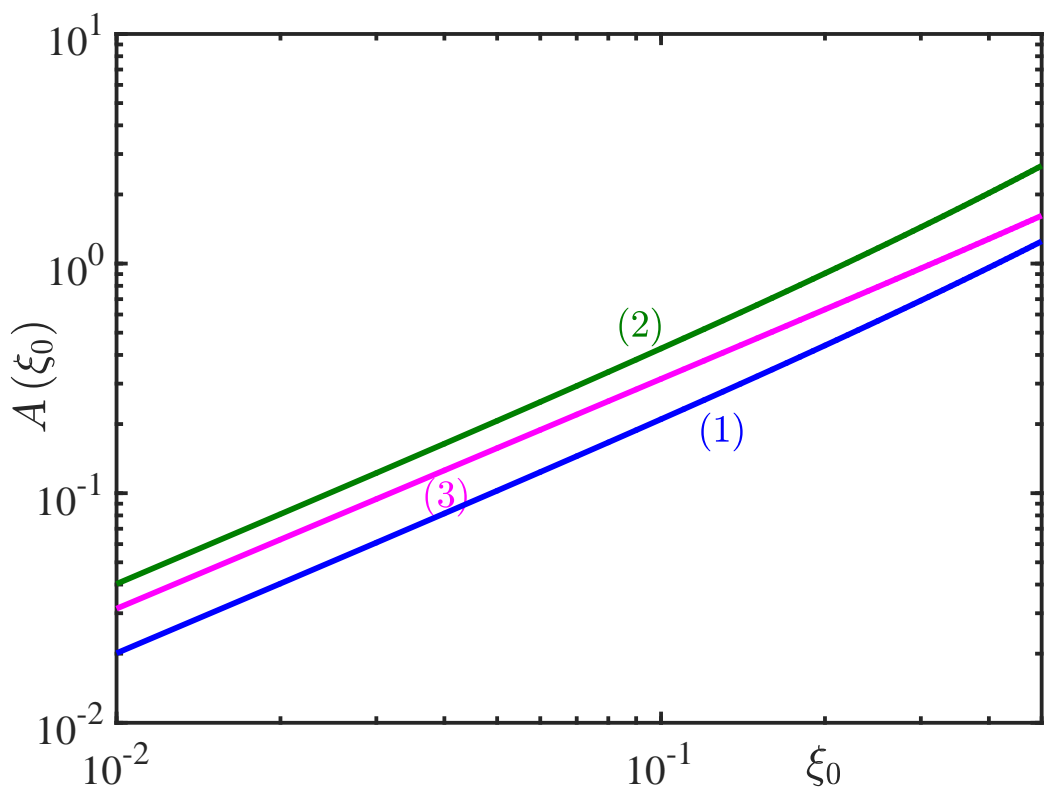

Fig. 1. (a) The 3 initial surface profiles: (1) Linear; (2) parabolic and (3) sinusoidal. (b) The function $A\left(\xi_{0}\right)$ vs $\xi_{0}$, that defines the minimal $\theta_{Y}$.

The functions $A\left(\xi_{0}\right)$ are plotted versus $\xi_{0}$ in Figure 1b, from which we conclude that the parabolic profile is most likely to move.

We observe from (43) that the minimal $\theta_{Y}$ is given by $\theta_{Y, \min }=B / A\left(\xi_{0}\right)$ and 
consequently the layer is static for

$$
1 \leq \frac{B}{A\left(\xi_{0}\right)}=\frac{\hat{L}_{0} \hat{\tau}_{y}}{\hat{h}_{0}^{2} \hat{\rho}(\hat{g}+\hat{a})} \frac{1}{\xi_{0} A\left(\xi_{0}\right)}
$$

This criterion can be understood physically as follows. The maximal inertial stress per unit area is $\left[\hat{h}_{0} \hat{\rho}(\hat{g}+\hat{a})\right]$. Due to the assumed mechanism of a dominant shear motion, this is scaled with a representative surface slope $\xi_{0} \hat{h}_{0} / \hat{L}_{0}$, to give a scale for the maximal generated shear stresses (multiplying again by $\left.A\left(\xi_{0}\right)\right)$. This is compared now with the yield stress, which it needs to exceed in order to flow.

\subsubsection{Spreading regimes}

Lets now assume that $B / A\left(\xi_{0}\right)<1$ so that there is some part of the oscillation during which the minimal $\theta_{Y}$ satisfies $\theta_{Y, \min }<1$ and there is at least some part of the oscillation for which the layer yields. Given the range of $\alpha(t)$ we see that as $\theta_{Y} \rightarrow 1^{-}$, we will have $\alpha(t)>0$, i.e. due to gravitational acceleration, $\alpha(t)>0$ during more of each oscillation than it is negative. We see that if $\chi \geq 1$ then $\alpha(t) \geq 0$ at all times and (41) remains parabolic. We call this regime, strong spreading, given that the spreading motion is always diffusive.

In contrast, if $B / A\left(\xi_{0}\right)<1$ and $\chi<1$ then for part of the oscillation we have $\alpha(t)<0$. We expect negative diffusion during this period if also $\theta_{Y}<1$, and label such flows unstable. To avoid the unstable regime we need to ensure that $\theta_{Y} \geq 1$ while $\alpha(t)<0$. The marginal condition is found to be when:

$$
\frac{B}{A\left(\xi_{0}\right)} \geq \frac{1-\chi}{\chi+1}
$$

Surfaces and parameters satisfying (49), for which also $\chi<1$ and $B<A\left(\xi_{0}\right)$, we classify as weak spreading. This has the physical meaning that although the net acceleration is upwards for a portion of the oscillation, the stresses generated during that part of the oscillation are insufficient to yield the fluid.

\subsubsection{Example numerical solutions}

To illustrate the spreading behaviours, we solve (41) numerically. For this we use a simple explicit finite difference method:

$$
\xi_{j}^{n+1}=\xi_{j}^{n}+\frac{\Delta t}{\Delta x}\left(q_{j-1 / 2}^{n}-q_{j+1 / 2}^{n}\right),
$$

where $\xi_{j}^{n}$ is the height of the free surface at time $t_{n}=n \times \Delta t$ and axial position $x_{j}=j \times \Delta x$. The timestep $\Delta t \propto \Delta x^{2}$ is selected to maintain stability, by 
estimating the maximum diffusivity in (41).

Our examples below all take the parabolic initial profile (2), which we saw was the most mobile, and fix $\xi_{0}=0.5$. First we show in Fig. 2 the results for frequency $f=1$, for both a strong $(\chi=\hat{g} / \hat{a}=2)$ and weak $(\chi=0.8)$ spreading regime. We mark the initial surface and that at $t=1 / f$, as well as selected intermediate profiles. The weak spreading profile has a slower evolution at the start and finish of the cycle (due to smaller $\alpha$ ), but similar evolution in the minimum of the cycle as $\alpha(t) \approx 1$ in both cases. Although the initial states are identical and the states at $t=1 / f$ are different (on close inspection), although they may be evolving to the same final state. The nonlinear nature of (41) is also evident, i.e. not all the surface spreads initially and the diffusivity is very local. For example, near the lowest parts of the surface the surface remains stationary until the (near linear) part of the profile propagates in from the outside, yielding the flow.

Figure 3 shows the same examples, except now for a higher frequency $(f=10)$. Again we see the slower evolution of the weak spreading case, particularly at start and finish of the oscillation, and the stationary surface at the lowest part. The main effect of increasing the frequency is a shortening of the time for evolution of the surface.

\subsection{Summary of flow regimes}

To summarise the flow regimes, we have identified the following:

(1) Static layer: if $\theta_{Y} \geq 1, \forall t$.

(2) Strong spreading: if $\alpha(t) \geq 0 \quad \forall t$ (found when $\chi \geq 1$ ), and $\theta_{Y}<1$ for part of the oscillation.

(3) Weak spreading: if $\alpha(t)<0$ for part of the oscillation, but only when $\theta_{Y} \geq 1$.

(4) Unstable: otherwise.

These regimes are illustrated in Fig. 4 , plotted in the $\left(\chi, B / A\left(\xi_{0}\right)\right)$-plane.

Working from the most stable regime to least stable, the regime boundaries are a little conservative in that the temporal aspect is not included explicitly. To clarify, $\alpha(t)=[\chi-\cos (2 \pi f t)] /[\chi+1]$ is initially minimal so might not move, whereas with a shift of time by half a period the spreading regimes will be driven by the maximal acceleration initially. 
(a)

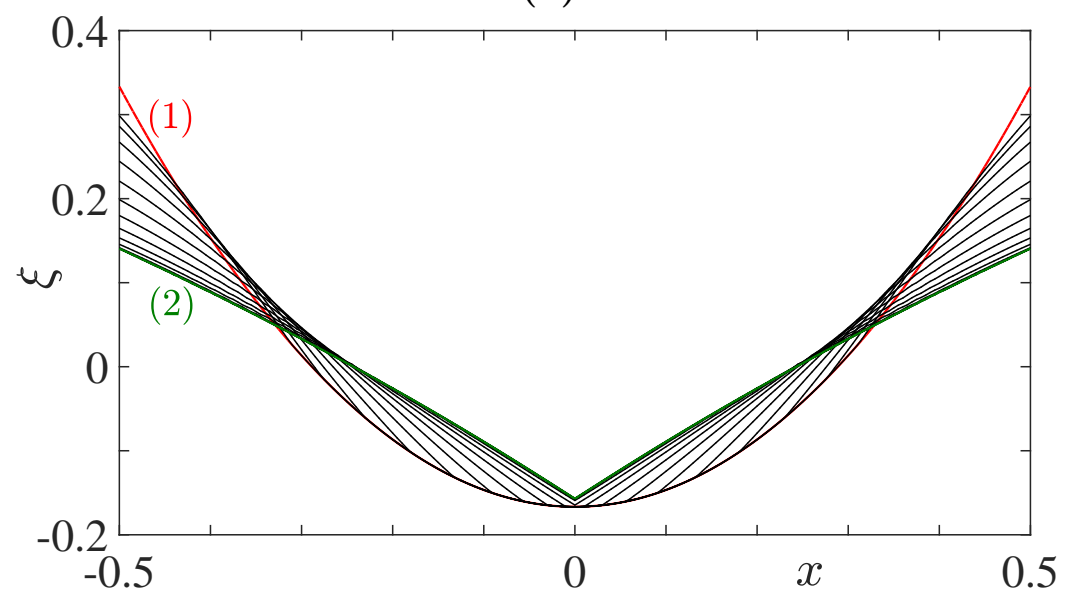

(b)

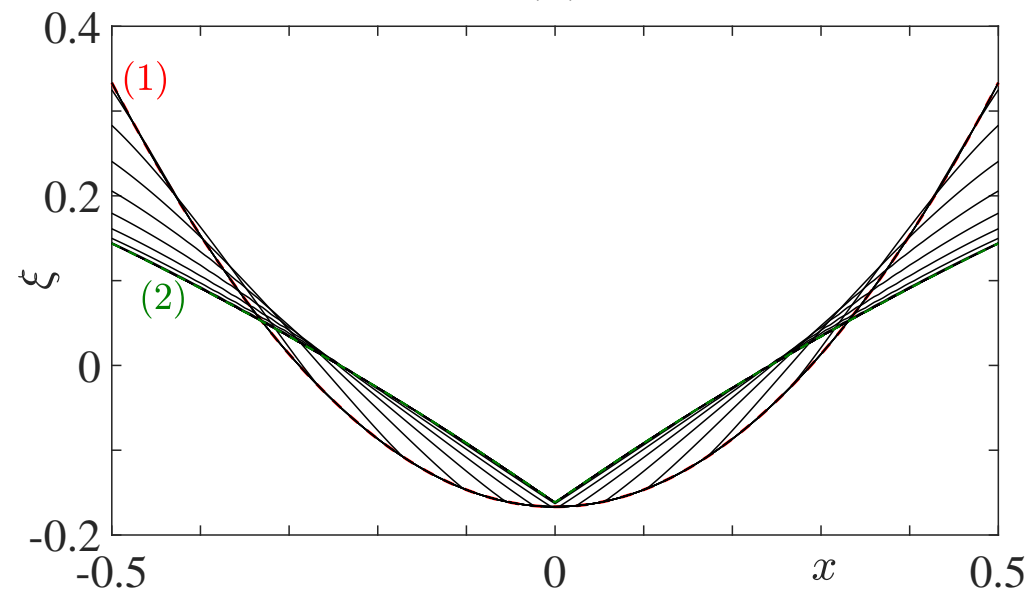

Fig. 2. Time evolution of the free surface in strong and weak spreading regimes at $f=1$ : a) strong spreading at $\chi=2$; b) weak spreading at $\chi=2$. For both we have $B=0.2 A\left(\xi_{0}\right)$ and have marked curves (1) and (2), which are the surface profiles at $t=0$ and $t=1 / f$, respectively.

\subsubsection{Final states}

A different temporal aspect is to note that all our stable spreading regimes appear to evolve towards a final regime that is static. Depending on the initial condition, it could be that some parts of the surface simply do not evolve at all, e.g. as the lower parts of the surface in Fig. 3 (although these may evolve at longer $t$ than that shown). Discounting this possibility and assuming that all parts of the surface move, we might return to (41) and examine when the spreading stops. It would appear to be as $\theta_{Y} \rightarrow 1^{-}$. As the spreading occurs whenever $\theta_{Y}<1$, the final interval of spreading occurs when $\alpha(t)=1$, from which we see that

$$
\left|\xi_{x}\right|(1+\xi)=B
$$


(a)

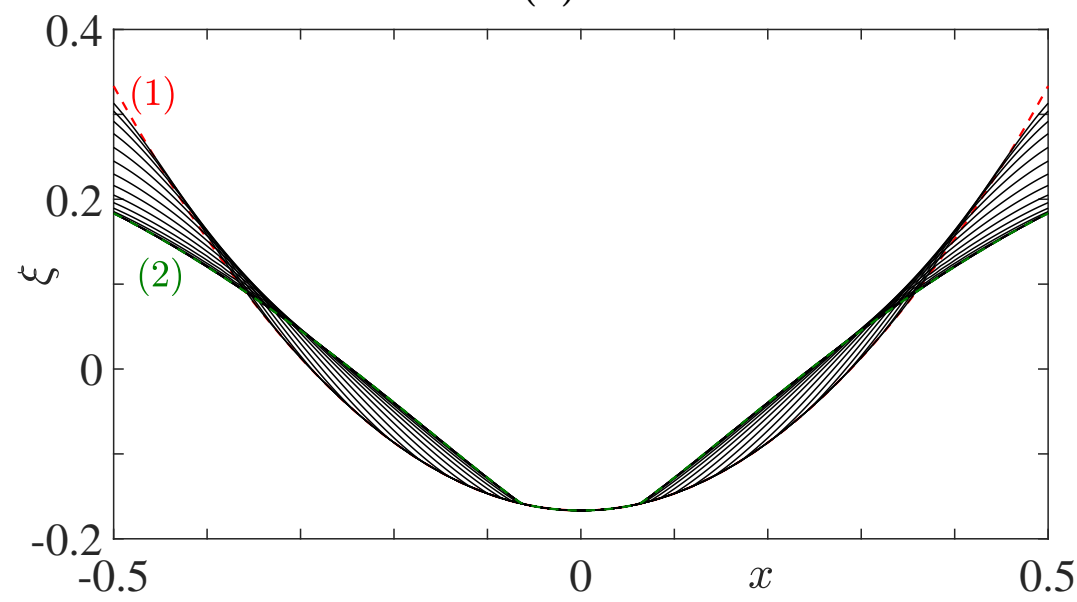

(b)

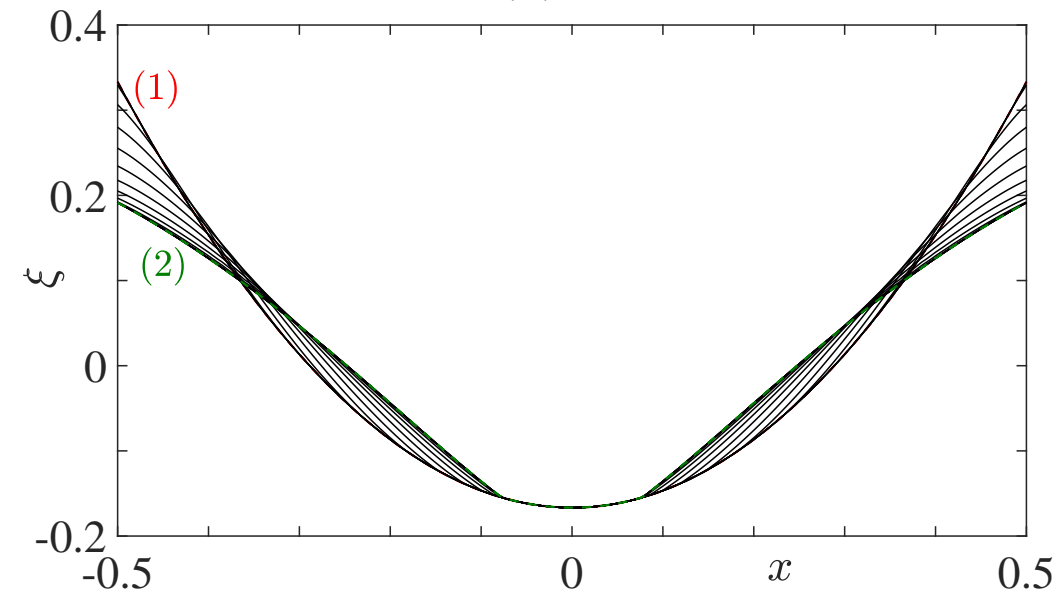

Fig. 3. Time evolution of the free surface in strong and weak spreading regimes at $f=10$ : a) strong spreading at $\chi=2$; b) weak spreading at $\chi=2$. For both we have $B=0.2 A\left(\xi_{0}\right)$ and have marked curves (1) and (2), which are the surface profiles at $t=0$ and $t=1 / f$, respectively.

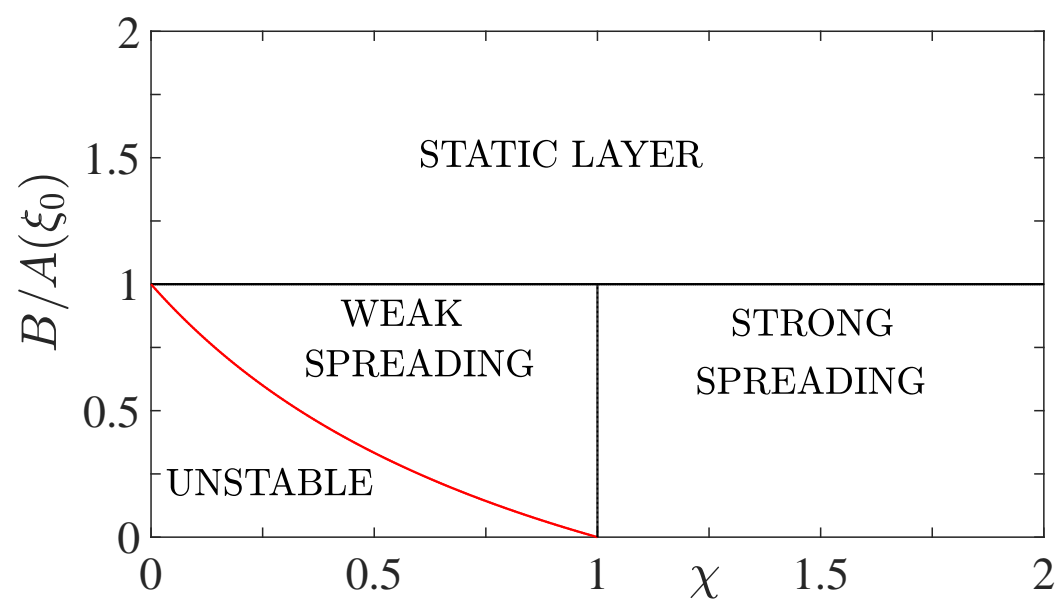

Fig. 4. The 4 different regimes identified. 
in this limit. Integrating this expression we find the final shape of the surface, say $\xi_{\infty}(x)$ :

$$
\xi_{\infty}(x)=\sqrt{2 B|x|+\left(\xi_{\infty}(0)+1\right)^{2}}-1 .
$$

The constant $\xi_{\infty}(0)>-1$ is to be found from the condition that the mean interface position is zero. As $B \rightarrow 0$ we find that $\xi_{\infty}(x)=0$. For small $B$ we find the approximation:

$$
\xi_{\infty}(x) \sim B(|x|-1 / 4)+O\left(B^{2}\right)
$$

We note the linear profile of the last computed shape in Fig. 2. The above analysis suggests that these may evolve to the same (approximately linear) final profile. Regardless of the small $B$ approximation, it is notable that $\xi_{\infty}(0)$ and hence $\xi_{\infty}(x)$ should depend only on $B$.

The above analysis is reminiscent of slump flow approximations and other predictions of final shapes: they are all conservative in the sense that they rely on surface motion continuing until the final shape is filled out, i.e. requiring that the fluid keeps moving until the final shape is achieved. At the same time in such problems, it is not hard to construct initial shapes that have segments where the slope is too shallow and that will remain stationary.

\subsubsection{Unstable flows}

Lastly we have some comments to make regarding the unstable flows. Principally, we wish to emphasize that the transition to unstable flows in Fig. 4 is not independent of the temporal features of the oscillation and represents only necessary conditions for instability. As we cross marginally into the unstable regime, the short period for which $\alpha(t)<0$ is balanced by a longer period for which $\alpha(t)>0$. It is possible that the diffusive spreading occurring during the period of positive $\alpha$ is sufficient to increase $\theta_{Y} \geq 1$. We have explored this feature numerically using different frequencies $f$ and different phase shifts of the oscillation, all close under the curve $B / A\left(\xi_{0}\right)=(1-\chi) /(\xi+1)$. We are able to find the above phenomenon marginally below this line, but the surface instability when it occurs is extreme and our simple explicit scheme is itself susceptible to numerical instabilities in these regimes. This makes numerical simulation the wrong tool for investigating further. An interesting avenue here could be to reintroduce the surface tension terms to stabilize the short wavelengths. 


\section{Discussion}

In this paper we have outlined a lubrication analysis of the typical Faraday instability scenario for a yield stress fluid layer. Our focus has been to work form the perspective of the stable static layer, understanding how yielding works and how stability is potentially restored during the spreading regimes. It is notable that the weak and strong spreading regimes that we have studied numerically evolve in one direction only: towards more stable and eventually static configurations. We have also derived the necessary conditions for instability at the limit of these spreading regimes.

Considering the experimental studies of $[17,18]$ we feel that our analysis and model may partly relate to the static layer states observed. We note however that in neither study is there any detail on control of the initial layer uniformity (critical here in our analysis), the aspect ratio of height to diameter is not typically asymptotically small and the discs/blobs placed on the oscillating plates have edges, where the surface gradients are necessarily large. However, new experiments could be performed in which the initial surface shape was explicitly molded.

Another difference here is with the lubrication model in [18], which does produce qualitatively reasonable pattern shapes. The model presented in [18] assumes a Newtonian viscosity for the gel, with a time dependency related to the acceleration. We feel this simplification misses some key features of the rheology, but so of course does an ideal visco-plastic fluid (as here). The type of separable model derived in [18] might conceivably result from a constitutive model of the sub-yield (low shear) behaviour as either a very viscous fluid or perhaps as a linear viscoelastic gel, although this is also not obvious to us. The model does not represent any generalised Newtonian fluid. In recent years there have been many constitutive models advanced to better explain the behaviour of hydrogels such as those in $[17,18]$, within these regimes. Evidently, the Faraday instability is a setup in which sub-yield behaviour could be critical in determining onset.

Our analysis simply sets the scene for what happens in the ideal case of a Hershel-Bulkley fluid. An interesting challenge is to better model the sub-yield rheological behaviour and/or to consider a different scaling for the dominant strain rates in a thin film model, i.e. compression/extension. We are considering this type of model as the next step. However, we feel that the analysis presented should be valid for suitably thin layers. 


\section{References}

[1] M. Faraday. On the forms and states assumed by fluids in contact with vibrating elastic surfaces. Phil. Trans. R. Soc. Lond, 121:319-340, 1831.

[2] T. N. Benjamin and F. Ursell. The stability of the plane free surface of a liquid in a vertical periodic motion. Proc. Roy. Soc. Lond. A, 225:505-515, 1954.

[3] K. Kumar and L.S. Tuckerman. Parametric instability of the interface between two fluids. J. Fluid Mech., 279:49-68, 1994.

[4] S. Douady and S. Fauve. Pattern selection in Faraday instability. Europhys. Lett., 6:221-226, 1988.

[5] S. Douady. Experimental study of the Faraday instability. J. Fluid Mech., 221:383-409, 1990.

[6] B. Christiansen, P. Alstrom, and M.T. Levinsen. Ordered capillary-waves states: quasicrystals, hexagons, and radial waves. Physical Review Letters, 68:2157, 1992.

[7] W.S. Edwards and S. Fauve. Patterns and quasipatterns in the Faraday experiment. J. Fluid Mech., 278:123-148, 1994.

[8] K. Kumar and K.M.S. Bajaj. Competing patterns in the faraday experiment. Phys. Rev. E, 52:R4606, 1995.

[9] A. Kudrolli and J.P. Gollub. Patterns and spatiotemporal chaos in parametrically forced surface waves: a systematic survey at large aspect ratio. Physica D, 97:133-154, 1996.

[10] F Raynal, S Kumar, and S Fauve. Faraday instability with a polymer solution. The European Physical Journal B-Condensed Matter and Complex Systems, 9(2):175-178, 1999.

[11] HW Müller and W Zimmermann. Faraday instability in a linear viscoelastic fluid. EPL (Europhysics Letters), 45(2):169, 1999.

[12] Pierre Ballesta and Sébastien Manneville. Signature of elasticity in the faraday instability. Physical Review E, 71(2):026308, 2005.

[13] Florian S Merkt, Robert D Deegan, Daniel I Goldman, Erin C Rericha, and Harry L Swinney. Persistent holes in a fluid. Physical review letters, 92(18):184501, 2004.

[14] Robert D Deegan. Stress hysteresis as the cause of persistent holes in particulate suspensions. Physical Review E, 81(3):036319, 2010.

[15] Claudio Falcón, Jake Bruggeman, Matteo Pasquali, and Robert D Deegan. Localized structures in vibrated emulsions. EPL (Europhysics Letters), 98(2):24002, 2012. 
[16] Daniel Bonn, Morton M Denn, Ludovic Berthier, Thibaut Divoux, and Sébastien Manneville. Yield stress materials in soft condensed matter. Reviews of Modern Physics, 89(3):035005, 2017.

[17] Hayato Shiba, Jori E Ruppert-Felsot, Yoshiki Takahashi, Yoshihiro Murayama, Qi Ouyang, and Masaki Sano. Elastic convection in vibrated viscoplastic fluids. Physical review letters, 98(4):044501, 2007.

[18] J.M. Wolf, S.R. Dungan, M.J. McCarthy, V. Lim, and R.J. Phillips. Vibrationinduced geometric patterns of persistent holes in carbopol gels. Journal of Non-Newtonian Fluid Mechanics, 220:99-107, 2015.

[19] GH Tattersall and PH Baker. The effect of vibration on the rheological properties of fresh concrete. Magazine of concrete research, 40(143):79-89, 1988.

[20] Boris N Ouriev and Naum B Uriev. Influence of vibration on structurerheological properties of a highly concentrated suspension. Measurement Science and Technology, 16(8):1691, 2005. 